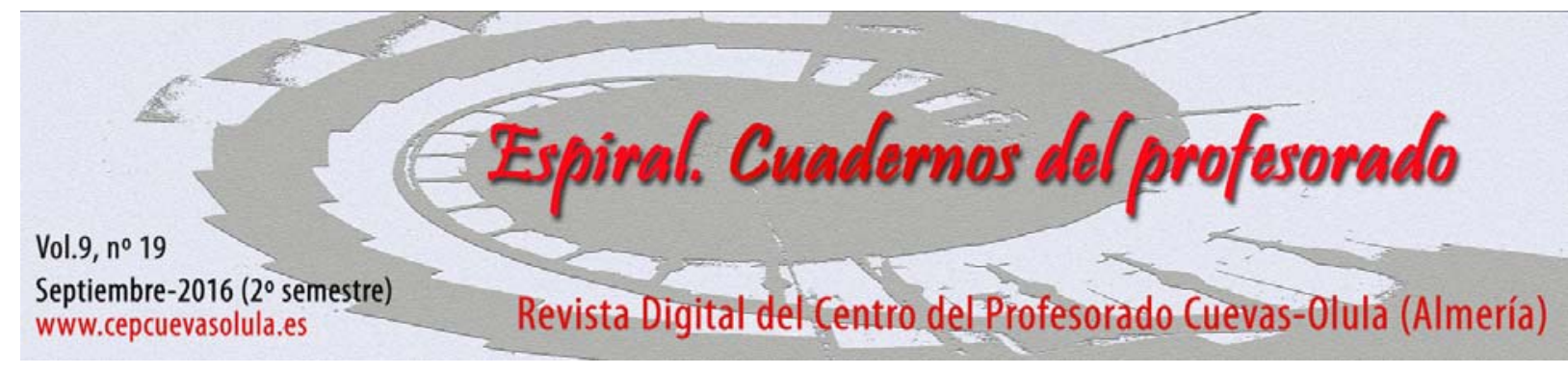

\title{
LAS TIC EN LAS PRÁCTICAS DE EVALUACIÓN EN EDUCACIÓN SECUNDARIA OBLIGATORIA
}

\author{
ICT in evaluation practices in secondary education
}

\author{
María de los Ángeles Jiménez Rodríguez
}

\author{
I.E.S. Puertas del Campo, Ceuta, España
}

\begin{abstract}
RESUMEN: La finalidad del proyecto es hacer explícita la necesidad de fomentar durante la práctica de evaluación del alumnado la integración de las TIC, pues favorece su motivación, regula su aprendizaje, y la coevaluación. De esta forma, los objetivos planteados es describir la práctica de la evaluación de los alumnos con las TIC y valorar su resultado. El estudio se enmarca en una metodología de naturaleza mixta. La muestra seleccionada para el estudio es un grupo específico de 15 alumnos de $2^{\circ}$ de E.S.O, un grupo de refuerzo de lengua castellana y todos de origen magrebí. Del total de alumnos de la muestra el 53\% son mujeres, frente al $47 \%$ restante que son varones, todos ellos con edades comprendidas entre los 14 y los 16 años, procedentes de un I.E.S de la Ciudad Autónoma de Ceuta. Esta experiencia puede hacerse extensible a institutos de similares características repartidos a lo largo de todo el territorio nacional. La autoevaluación presencial en el aula a través del apoyo de distintos recursos TIC, resultó una experiencia positiva y motivadora para el alumnado, mostrando un gran interés por medir el logro de sus conocimientos y seguir mejorando académicamente.
\end{abstract}

Palabras clave: Evaluación, Recursos TIC, Regulación enseñanza -aprendizaje, coevaluación y Motivación.

ABSTRACT: The aim of the project is to make explicit the need to promote during the practice of student assessment integration of ICT, it favors motivation, regulates learning and peer assessment. Thus, the objectives describe the practice of assessing pupils with ICT and assess its outcome. The study is part of a methodology of mixed nature. The sample selected for the study is a specific group of 15 students of 2 nd E.S.O, a group of reinforcement of Spanish language and all of North African origin. Of the students sample $53 \%$ are women, compared to the remaining $47 \%$ are males, all aged between 14 and 16 years, from a I.E.S of the Autonomous City of Ceuta. This experience can be extended to similar institutes scattered throughout the country. The face self-assessment in the classroom through support of different ICT resources, was a positive and motivating experience for students, showing great interest in measuring the achievement of their knowledge and continue to improve academically.

Key words: Evaluation, ICT Resources, Regulation teaching-learning, peer assessment and Motivation.

Jiménez Rodríguez, M. A. (2016). Las TIC en las prácticas de evaluación en Educación Secundaria Obligatoria. Espiral. Cuadernos del Profesorado, 9(19), 56-67. Disponible en: http://espiral.cepcuevasolula.es/

Fecha de recepción: 28/05/2016

Fecha de aceptación: 23/07/2016
Enviar correspondencia a: woman167167@hotmail.com

\section{Introducción}

La principal necesidad a la que se pretende dar respuesta a la necesidad de fomentar la motivación en alumnos(as), en su mayoría de origen magrebí, provenientes de familias de escasos recursos económico, bajo rendimiento académico y nulo interés hacia el aprendizaje de la materia de 
lengua castellana. Por lo tanto, el tema objeto de estudio es describir una experiencia y conocer la actitud del alumnado desde su propia implicación en la evaluación de sus conocimientos a través de las TIC y valorar las ventajas de esta variación en la metodología de la evaluación.

El proyecto plantea aprovechar el potencial de las TIC, ya que es innegable la eficacia de las TIC en la educación, pues son múltiples las experiencias y trabajos de investigación que lo avalan. Además, son múltiples las herramientas, que nos permite difundir conocimientos, pero a su vez también hay otras herramientas de apoyo al proceso de evaluación.

La evaluación es un factor esencial que permite valorar el aprendizaje de los estudiantes, e incidir en un cambio de metodología en la construcción del aprendizaje para obtener resultados óptimos, pero ¿por qué no también hacer uso de esta nueva tecnología en el proceso de evaluación del alumnado?

Es por ello, interesante revisar la literatura para conocer diversos sistemas de evaluación del aprendizaje de los estudiantes que resulten motivadores. Aunque, actualmente podemos afirmar que todavía hay una escasa tradición en el uso de las TIC para la evaluación de los procesos de enseñanza y aprendizaje. Las estrategias evaluadoras más tradicionales siguen estando en alza, son frecuentes el diseño y aplicación de pruebas objetivas y exámenes. Las TIC ofrecen posibilidades para diseñar múltiples instrumentos de seguimiento; organizar la información recogida en el proceso evaluador e interpretarla, facilitando así la comprensión del proceso de aprendizaje, en algunos casos al docente, en otros directamente a los estudiantes y en otros a ambos.

Como señalan García Carrasco y otros (2002), la evaluación es imprescindible en el proceso de enseñanza-aprendizaje, como mediada para obtener la consecución de los objetivos de aprendizaje por parte del alumno, y, también, como control de la calidad de dicho proceso.

Lara $(2001,2003)$ se han encontrado gran cantidad de investigaciones y de herramientas, en continuo desarrollo, para gestionar la evaluación a través de tecnología web. A nivel superficial, se pudo observar que estos programas informáticos que sirven para evaluar el rendimiento de los alumnos a través de Internet se pueden clasificar en tres categorías básicas:

1. Entornos virtuales de formación (web-based trining), que ayudan al profesor a gestionar un módulo o curso de enseñanza completo a través de la red (distribución de contenidos, intercambios con los alumnos a través de correo electrónico, foros de discusión o chats y evaluación de los alumnos). Ejemplos de este tipo: WebCT, Learning Space, Edustance, etc.

2. Herramientas de autor, consisten en software destinado a su vez a la creación de programas a modo de ejercicios o tareas. Ejemplos de este tipo son: Hot Potatoes, Quia!, Clic, etc.

3. Software específico más complejo (requiere manejo de servidor), que permite poner en la red a toda la institución, tanto para la creación y publicación de los exámenes, como para recoger los resultados de los estudiantes. Ejemplos de este software son: Perception y Quiz Factory.

Charman (1999) señala cuáles pueden ser las principales ventajas del uso de evaluación a través de Internet:

- Frecuencia de la evaluación

$\checkmark$ Inmediato feedback (a los estudiantes les interesa recibir la retroalimentación ya que enlazan su producción con los resultados, se les ofrecen ayudas, orientaciones y produce todo ello motivación para seguir estudiando)

$\checkmark$ Inmediata corrección de los trabajos por los profesores (de modo que los errores puedan ser detectados $\mathrm{y}$, si es necesario, adaptar ciertos contenidos)

$\checkmark$ Fiabilidad en la medición (estabilidad, precisión), debido al tipo de pruebas (objetivas) que se suelen utilizar.

$\checkmark \quad$ Flexibilidad de acceso (espacio y tiempo) 
De todos los estudios sobre evaluación a través de la red se puede concluir que es beneficiosa, tanto para el estudiante como para el profesor. A los alumnos les sirve para mejorar su nivel de competencia, les motiva para el estudio y, en definitiva, resulta una herramienta útil para su aprendizaje. Junto a ellos, los profesores valoran la facilidad de poder enviar al alumno un feedback inmediato a través de la elaboración de comentarios u orientaciones que guían su aprendizaje. No obstante, se debe tener en cuenta como sostiene (Taras, 2003) la calidad del feedback, es decir en utilizar en la evaluación en su modalidad formativa información detallada de su actuación.

Por último la motivación constituye, así mismo, uno de los principales pilares de la educación. El estudiante motivado se involucra y concentra más en la clase y ello favorece el aprendizaje (Passey et al. 2004, en Condie y Munro, 2007). Aún más, la experiencia de algunos programas de informática educativa ha mostrado que el aumento de la motivación de los estudiantes por el uso de las TIC en clases aumenta el nivel de asistencia al colegio (Borthwick y Lobo, 2005). Se ha demostrado que la naturaleza visual de algunas tecnologías involucra más a los estudiantes y refuerza la comprensión de conceptos (Livingstone y Condie, 2003; Passey, 2004; citados en Condie y Munro, 2007).

En definitiva la premisa común de todos los investigadores es que aluden a la eminente necesidad de producir cambios e innovaciones en la evaluación apoyados en las TIC, del mismo modo que se vienen produciendo cambios en las formas de enseñar y aprender.

\section{Origen de la experiencia y objetivos}

Nuestro análisis previo ha evidenciado resultados académicos negativos de un grupo de 15 alumnos(as) de $2^{\circ}$ E.S.O, con refuerzo en lengua castellana. El alumnado manifiesta escaso interés por la materia, ya que la consideran poco motivadora, además en sus casas no la utilizan, si no el dariya como lengua materna, pues la mayoría son musulmanes lo que da lugar a un escaso interés por dominar plenamente la lengua castellana.

Por tanto, consideramos la necesidad de contribuir a la mejora de la práctica de la evaluación del alumnado, a través de las TIC, y que propicie un óptimo aprovechamiento de los diferentes recursos tecnológicos y didácticos, así como el empleo de estrategias que potencien la evaluación y colaboración entre los sujetos participantes del entorno virtual, ya que, su tendencia consiste en disminuir las dificultades de los jóvenes en cuanto a su aprendizaje de la Lengua.

Una vez mostrado el contexto y la fundamentación teórica de la investigación, pasaremos a definir las preguntas de investigación surgidas durante el desarrollo del proyecto y que nos llevaron a definir los objetivos de la presenta investigación.

Tras lo expuesto en capítulos anteriores acerca de la importancia y relevancia del uso de las TIC en el aula, así como la importancia de la motivación en nuestros alumnos(as), se plantean las siguientes preguntas de investigación:

1. ¿El uso de ambientes enriquecidos por las TIC desde la práctica de la evaluación, incrementa la motivación y favorece un aprendizaje formativo?

2. ¿Este tipo de metodología me permite adaptarme mejor a las necesidades de los alumnos?

En consonancia con las anteriores preguntas de investigación se plantea el siguiente objetivo general para este proyecto de investigación:

- Objetivo: Describir y relacionar que la práctica de la evaluación del alumnado apoyado en las TIC motiva la autoregulación de su aprendizaje y la coevaluación. Este objetivo general se concreta en tres objetivos específicos:

- Objetivo 1: Evaluar si el docente ha podido descubrir las ventajas de la incorporación de las TIC en la práctica de la evaluación del alumnado.

- Objetivo 2: Averiguar si la implicación en la práctica de la evaluación del alumnado permite la autoregulación de su aprendizaje y la coevaluación.

- Objetivo 3: Medir si el alumnado muestra deseos de aprender con esta práctica. 


\section{Metodología}

La metodología aplicada a este proyecto es mixta, un enfoque cuantitativo y cualitativo. Los pilares sobre los que sustenta este enfoque mixto son los siguientes:

- Es un proyecto inductivo: en el que partimos de experiencias determinadas para extraer conclusiones generales.

- Es un proyecto en el que utilizamos los datos obtenidos sin incorporar nuestros conocimientos previos.

- Es un proyecto que estudia la realidad de un centro educativo concreto.

Esta experiencia se ubica en un I.E.S ciudad autónoma de Ceuta. Un alto porcentaje del alumnado es de origen magrebí, provenientes de familias de bajos recursos económicos y bajo nivel académico. La mayoría de ellos no tiene un interés por su futuro y por alcanzar estudios superiores.

Esta experiencia surge desde nuestra exigencia por lograr la motivación de los alumnos por aprender. La muestra del caso responde a 15 alumnos(as) de $2^{\circ}$ curso de la E.S.O del I.E.S. Puertas del Campo (Ceuta), de escasa motivación por su aprendizaje.

Para lograr en lo posible la motivación por aprender, hemos procedido a la variación de la tradicional metodología en el proceso de evaluación del alumnado, apoyándonos en las TIC, que propicia un proceso de autoregulación del aprendizaje, donde los alumnos son capaces de valorar lo aprendido o no, y si desarrolla correctamente las tareas o no. Por otro lado, la puesta en práctica de la coevaluación en el aula, permite que todos puedan evaluar. La evaluación se concibe así como una responsabilidad no exclusiva del profesorado.

Tabla 1

Cronograma de las sesiones

\section{CRONOGRAMA DE LAS SESIONES}

MAYO 2015

$\begin{array}{llll}1 & 12 & 14 & 28\end{array}$

CLASE 1

$\mathbf{X}$

Explicación de la actividad. Toma de contacto con el proyecto y con la plataforma Socrative

CLASE 2

Cuestionario de los contenidos de la materia LC.

CLASE 3

Repetición de los cuestionarios afianzar los conocimientos, a través de la sección juegos en grupo.

CLASE 4

Cuestionario de Comprensión lectora

Una vez planteada nuestras objeciones que nos encontramos en el aula, y justificado la incorporación de los recursos TIC en nuestra práctica docente desde la fase de la evaluación de los alumnos, daremos comienzo a la descripción del proceso de investigación qué consiste en la implementación de los recursos TIC en el proceso de evaluación.

La planificación de la experiencia se muestra en el siguiente cronograma del desarrollo de las sesiones y actividades del proyecto, tras la finalización de cada unidad didáctica, que aparece recogido en la Tabla 1 y Tabla 2 .

En todo momento se ha recurrido a la observación directa de los alumnos, así como a otros instrumentos específicos de recogida de información, como son: el test de evaluación desde Socrative, el diario y el cuestionario final, que pasamos a detallar.

- El test de evaluación desde la plataforma Socrative: herramienta c on la que se pretende medir el nivel de conocimientos adquiridos tras finalizar una unidad didáctica y, al mismo tiempo, motivar al alumnado en el seguimiento y realización de actividades. 
El entorno virtual elegido para promover la autoevaluación del alumnado es la plataforma de evaluación online Socrative, a través de la cual el alumno(a) descubrirá el conocimiento real que tiene de los contenidos global de la unidad, de forma autónoma, motivadora, y a la vez enriquecedora, pues el conocimiento inmediato de los resultados, favorece la reorientación del conocimiento en positivo. Se favorece así un aprendizaje muy motivador.

Destacar que Socrative es una plataforma de evaluación-online, que permite nuestro propia autoevaluación de forma inmediata, utilizando un interfaz sencillo y un diseño de actividades de autoevaluación bastante atractivas. Además, es una aplicación que genera actividades para todo tipo de dispositivos móviles (portátiles, tabletas o smartphones) con el objetivo de involucrar activamente al alumnado en clase, permitiendo aumentar la motivación y participación de los discentes en su aprendizaje.

Tabla 2

Desarrollo de la sesiones

\section{DESARROLLO DE LAS SESIONES}

CLASE 1 En este primera sesión se realizará una descripción de la plataforma de seleccionada, Socrative, para llevar la práctica de la evaluación del alumnado, explicándoles a los alumnos(as) el funcionamiento de la herramienta, a través de la visualización de un vídeo explicativo, y su puesta en práctica. Se realiza la evaluación de los contenidos aprendidos de la unidad didáctica al finalizar ésta. Se establecerá en cada unidad didáctica, que tiene una duración de dos semanas, los siguientes bloques que serán evaluados en el aula virtual, antes del examen oficial:

Bloque 1. "El género poético"

Bloque 2. "Los recursos estilísticos"

Bloque 3. "sintaxis"

Bloque 4. "ortografía"

Bloque 5. "Lectura comprensiva".

CLASE 2 En esta se realiza la evaluación del alumnado de los contenidos explicados al finalizar la unidad didáctica de forma individual.

CLASE 3 En esta sesión se repetirán la evaluación de los contenidos explicados de la unidad didáctica, de forma grupal, a través de la sección de juegos, la participación será en grupo, con la ayuda de los compañeros, para que superen definitivamente los errores del test anterior, de cara al examen final. Las competiciones a través de la sección de juegos "carreras espaciales", es un espacio motivador y que crea competitividad y granas de esforzarse entre ellos. Además de fomentar el diálogo y el debate entre ellos.

CLASE 4 Durante la semana, se dedicará un día a la semana al fomento de la lectura. Para ello, se desarrollarán a través de un soporte motivador que ofrece el centro y de fácil manejo; E-book. Con la intención de motivarles, al final de la lectura marcada para ese día por el tutor-docente, se realizará igualmente una actividad de evaluación de los contenidos de la lectura de comprensión. Se realizarán igualmente de forma individual o en grupos, a partir de una sección muy motivadora, como es la sección de juegos.

Durante la implementación del proyecto no ha sido necesario la ubicación en un aula específica, se ha hecho uso de su aula cotidiana, tan solo se ha requerido la adjudicación de una contraseña de acceso a la wifi del aula, los alumnos han traído su propio instrumento TIC, móvil o Tablet, para proceder al desarrollo de las actividades de evaluación online diseñadas en la plataforma Socrative, una plataforma de evaluación online, proyectada en la pizarra digital del aula el cuestionario.

En todo momento, el profesor guía el proceso de conexión a la plataforma www.socrative.com, a la cual se le accede a través de la contraseña aportada por el profesor, en este caso "AULAMARIA", a partir de este paso, cuando todos los alumnos hayan metido su clave de acceso "AULAMARIA", el profesor da paso al inicio de las actividades de evaluación. El test de 
evaluación ha sido diseñado por el profesor desde la propia plataforma, variando el formato de la pregunta y respuestas que brinda la plataforma; tales como, preguntas con respuesta de verdadero o falso, preguntas con respuestas cortas, preguntas con respuestas múltiples.

El alumnado, tras acceder con la contraseña al aula de evaluación virtual "AULAMARIA", procedieron a la realización del cuestionario, elaborado previamente por la profesora, y que a ellos le aparecen las preguntas de una en una, siendo necesario contestarla para pasar a la siguiente, y en la pizarra digital se proyectará el nombre de los participantes con el cuestionario y la evolución de los porcentajes de los alumnos en función de la contestación de sus preguntas. Además aparecerá proyectado en la pizarra digital, la opción acertada de las preguntas, como aparece en las Figuras 1 y 2 , o bien ocultando sus respuestas para que los demás compañeros no se sirvan de los resultados de los compañeros, Figura 3.

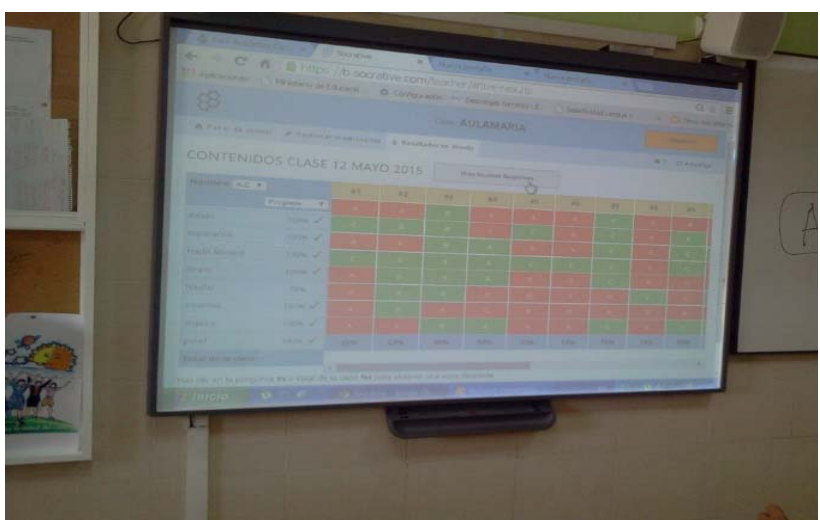

Figura 1. Fotografía en el aula de la evaluaciónonline realizada por los alumnos de los contenidos sin ocultar las respuestas de la unidad didáctica en la clase del 12 de mayo 2015.

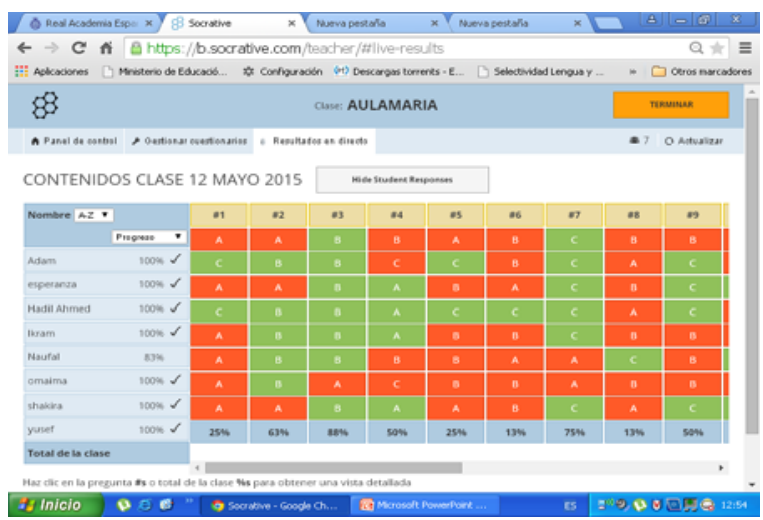

Figura 2. Captura de pantalla del test de la evaluación-online realizada por los alumnos de los contenidos sin ocultar las respuestas.

La proyección del test de evaluación en la pizarra digital, permite que los alumnos adopten una actitud competitiva por alcanzar antes que los demás el 100\% del cuestionario, además permite al docente y a los alumnos conocer en tiempo real y global sus errores y aciertos, tanto los suyos o del resto de sus compañeros. Los números de errores hasta llegar al acierto serán registrados por la aplicación de Socrative, y a través de la obtención de los resultados en formato EXCEL como se observa en la Figura 4, se observará todos esos detalles, lo que permite al docente saber cuál ha sido la pregunta más fallida, lo que permite al docente incidir en ese concepto evaluado previo al examen escrito de los alumnos. Además, la plataforma permite generar los resultados en forma de estadísticas de la evolución de todo el grupo.

Otra estrategia que permite



Figura 3. Captura de pantalla de los resultados de un test elaborados por los alumnos ocultando las respuestas solo apareciendo el tanto por ciento de aciertos de cada uno del cuestionario y el porcentaje de aciertos de cada uno.

la plataforma y que se ha puesto en práctica para la realización de los cuestionarios en formato online, es a partir del formato de juegos que brinda la plataforma Socrative que denominada "carreras 
espaciales" y que permite que los cuestionarios sean realizados en grupo, fomentando así el aprendizaje colaborativo. Los alumnos irán eligiendo su grupo a partir de la elección de un color, con un icono elegido por la profesora, en este caso la imagen de una bicicleta, como se puede observar en la imagen de la Figura 5 y 6.

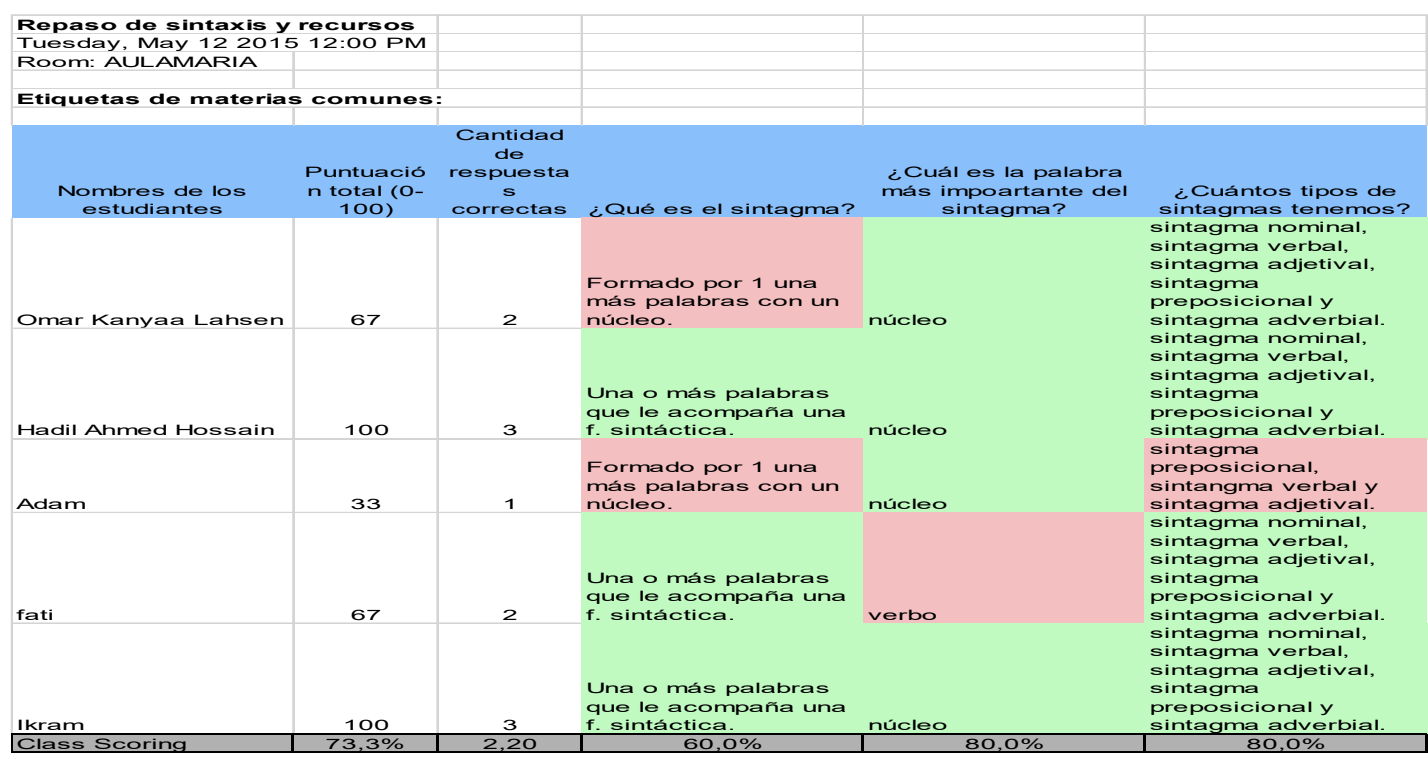

Figura 4. Captura de pantalla de un fragmento de la hoja Excel que brinda la opción la plataforma Socrative, se observa el nombre del estudiante, y la puntuación obtenida.

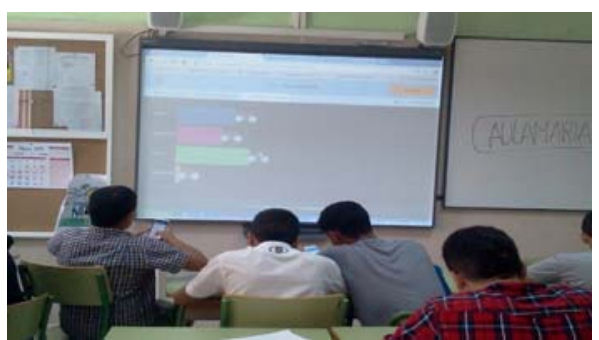

Figura 5. Pizarra digital donde se proyectaba una de las "carreras espaciales", realización del cuestionario en grupo en forma de juego, otra posibilidad que ofrece la plataforma "Socrative".



Figura 6. Captura de pantalla donde se proyectaba una de las "carreras espaciales", realización del cuestionario en grupo en forma de juego, otra posibilidad que ofrece la plataforma "Socrative".

- El diario de clase, recoge las siguientes observaciones: el funcionamiento de la herramienta Socrative, la detección de problemas técnicos, las destrezas de los alumnos, la capacidad de trabajar con los diferentes recursos, la competencia digital del alumnado, la motivación de los mismos y, finalmente el uso de los recursos específicos de aprendizaje empleados.

- El cuestionario final para los alumnos(as) implicados: Está formado por un total de 21 ítems, 17 de los cuales se corresponden a preguntas cerradas tipo Likert con cinco respuestas (TD, D, I, A, TA) y los cuatro restantes a preguntas abiertas en las que los alumnos(as) participantes pueden expresar su opinión y aportar sus propios puntos de vista. Las siglas anteriores se corresponden a los siguientes significados: TD (totalmente en desacuerdo); D (en desacuerdo), I (indiferente); A (de acuerdo); TA (totalmente de acuerdo). La Tabla 3 muestra el objeto de estudio de cada uno de los ítems empleados en el cuestionario, agrupados por categorías. 
Tabla 3

Categorías del Cuestionario Final. Fuente: Elaboración propia

\begin{tabular}{cll}
\hline ITEMS & \multicolumn{1}{c}{ DIMENSIÓN } & \multicolumn{1}{c}{ OBJETO DE ESTUDIO } \\
\hline [1-5] & $\begin{array}{l}\text { Conocer la relación de las } \\
\text { TIC en el proceso de } \\
\text { aprendizaje. }\end{array}$ & $\begin{array}{l}\text { Son preguntas enfocadas a la relación del } \\
\text { alumnado con el uso de las TIC como } \\
\text { herramientas facilitadoras de dicho aprendizaje. }\end{array}$ \\
& $\begin{array}{l}\text { Conocer las necesidades del } \\
\text { alumnado en la materia de } \\
\text { L. Castellana. }\end{array}$ & $\begin{array}{l}\text { Son preguntadas dirigidas a averiguar si este tipo } \\
\text { de experiencia ha llevado a los alumnos(as) a } \\
\text { mejorar su relación con la materia, a acercarse más } \\
\text { a ella, a dedicarle más tiempo, a disfrutar haciendo } \\
\text { las actividades. }\end{array}$ \\
& $\begin{array}{l}\text { Son preguntas dirigidas a conocer si los } \\
\text { alumnos(as) se habían divertido. }\end{array}$
\end{tabular}

\section{Resultados y repercusión}

En este proyecto utilizamos el método de triangulación de datos de forma personal, y que integran los siguientes instrumentos de evaluación; test de evaluación, el diario y el cuestionario. Mediante la implementación de esta técnica analizaremos los datos obtenidos, y así poder obtener las conclusiones y recomendaciones adecuadas en consonancia con el objetivo general de este proyecto de investigación: "Describir y relacionar que la práctica de la evaluación del alumnado apoyado en las TIC motiva la autoregulación de su aprendizaje y la coevaluación." Este objetivo general que a su vez se concreta en tres objetivos específicos, y que establecemos su relación con los métodos de investigación en la siguiente Tabla 4.

\section{Tabla 4.}

Relación entre objetivos y métodos de investigación. Fuente: elaboración propia.

HIPÓTESIS GENERAL: La práctica de la evaluación del alumnado apoyado en las TIC; motiva la autoregulación de su aprendizaje y la coevaluación

Objetivos/hipótesis
Diario

Test Evaluación
Cuestionario

1. El docente ha podido descubrir las ventajas de la incorporación de las TIC en la práctica de la evaluación del alumnado.

2. La implicación en la práctica de la evaluación del alumnado permite la autoregulación de su aprendizaje y la coevaluación, en la materia, L. Castellana.

3. El alumnado muestra deseos de aprender con esta práctica.

X X X

$\mathrm{X} \quad \mathrm{X}$

$\mathrm{X} \quad \mathrm{X}$

Los resultados recogidos a través de los diferentes instrumentos, son los siguientes:

- Resultados del test de evaluación desde la plataforma Socrative: los alumnos procedían a realizar las actividades de evaluación diseñadas en el entorno virtual Socrative. Cada una de estas pruebas se correspondía a los contenidos practicados a lo largo de la semana presencialmente en clase, y luego serían evaluados por ellos mismos, desde una metodología lúdica y atractiva. Las pruebas están formadas por una media de 10 preguntas tipo test, incluyendo algunas de ellas 2 o 3 preguntas, en las que se les proporciona alternativa de elección a los alumnos(as). Han sido realizadas para responderlas con una duración estimada de 5 minutos. Con esta herramienta se mide el nivel de conocimientos adquiridos durante la clase y, al mismo tiempo, motivar al alumnado en 
reorientar su aprendizaje en positivo, a través del conocimiento inmediato de sus errores. Este instrumento de recogida de información se ha considerado determinante en esta experiencia, pues muestra en tiempo real las puntuaciones de los alumnos, así como número de intentos hasta conseguir el acierto, como se muestra anteriormente en las Figuras 3 o 4. Por otro lado, si se quiere un análisis más detallado se solicita a través de la sección de informe, que ofrece tres opciones: obtener informe en hoja Excel, visualización de una gráfica, e ir al Panel de control, como aparece en las Figuras 7 y 8.

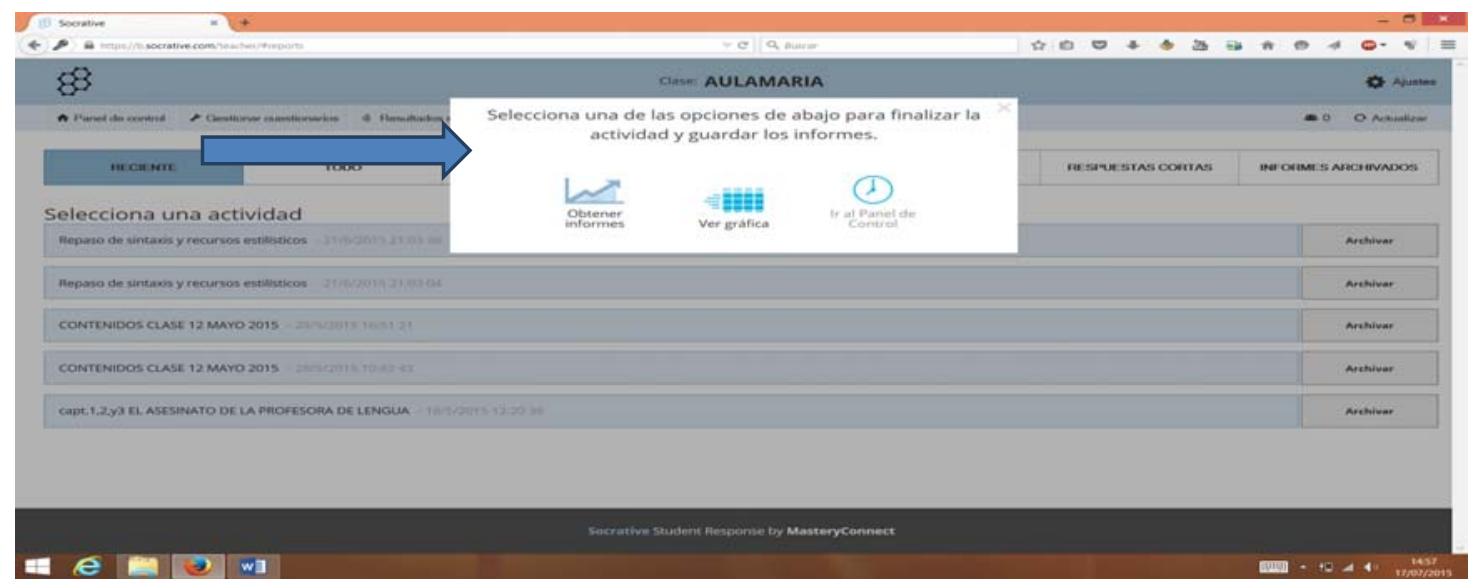

Figura 7. Sección de Informes para la obtención de los resultados de los test.

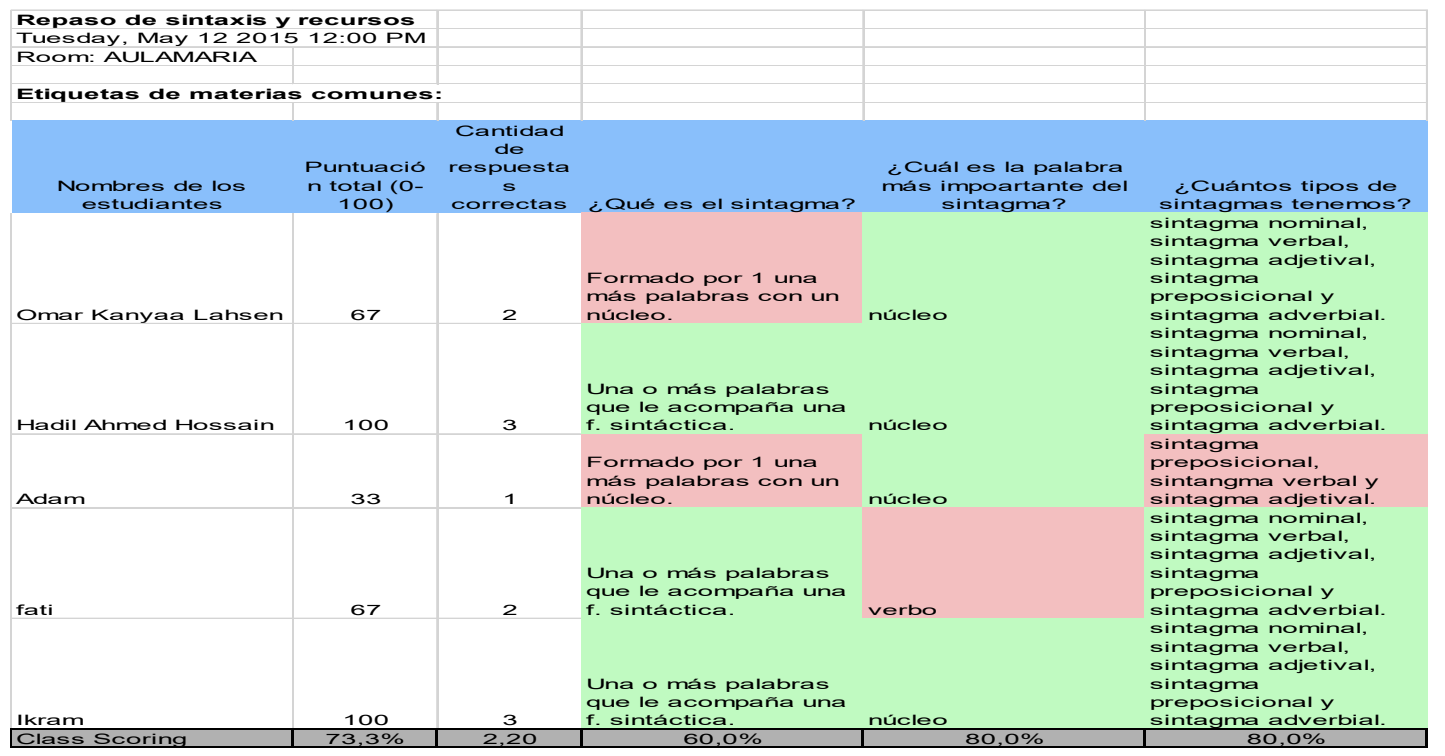

Figura 8. Porcentajes de las puntuaciones obtenidas por los alumnos y la cantidad de respuestas correctas.

- Resultados extraídos del Diario: La información recogida en el diario a lo largo de todos los días de proyecto ha sido determinante en el análisis de resultados. En este punto se describen de forma detallada cada una de las categorías analizadas en el análisis y estudio de dicho diario:

- Incidencias técnicos. El diario refleja distintos tipos de incidentes relacionados con esta categoría. El número de alumnos en total 15 no se corresponde con el número de móviles para llevar a cabo la práctica de la evaluación, pues se observa un limitado número de móviles. Por lo tanto, no todos se responsabilizaron de traer su propio soporte TIC para desarrollar la autoevaluación de su aprendizaje, lo que contribuyó a 
que los alumnos estuviesen incómodos en algunas ocasiones y no pudiesen realizar las actividades de manera individual.

- Competencia digital. La experiencia ha demostrado que la mayoría de los alumnos posee poca destreza en el uso de los recursos digitales, requiriendo una constante atención por parte del profesor. En otros casos, los compañeros(as) contiguos se ofrecían para ayudar a aquellos con menos destreza en el manejo del dispositivo móvil que contribuye al desarrollo de la evaluación del alumnado desde la plataforma Socrative.

- Acceso a la plataforma. Los alumnos no presentaron ninguna dificultad para acceder a la plataforma.

- Destreza de los alumnos en el uso de los recursos empleados. Los recursos propuestos a través de la plataforma Socrative son bastante sencillos de utilizar en general, aunque algunos de ellos necesitaron de una explicación generalizada de su funcionamiento. Y, sobre todo la óptima destreza en el uso del móvil, un recurso TIC con el que se encuentran muy familiarizados.

- Motivación del alumnado. A partir de la lectura de dicho diario y de la observación directa por parte del profesor, se detecta una clara motivación del alumno(a) y predisposición hacia el uso de este tipo de metodología. Del mismo modo, se observa cómo los alumnos, deseosos de conocer la puntuación de su conocimiento, y sobre todo la mayor puntuación, generan entre ellos un espíritu de competitividad y de esfuerzo. Ninguno de los quince alumnos, pese a las dificultades de algunas preguntas, las dejan de lado, y se mostraban concentrados durante todo el proceso.

- Funcionamiento de Socrative. El uso de la herramienta Socrative lo describiría como altamente satisfactorio. Los alumnos no tuvieron ningún problema en familiarizarse con el uso de esta herramienta y accedían a ella fácilmente.

- Resultados e x t r a íd os del cuestionario final: el cuestionario final fue respondido por un total de 15 alumnos y han sido analizadas cada una de sus preguntas de forma individual, así como agrupadas por bloques de contenidos:

- En el primer bloque de contenidos (conocer la relación de las TIC en el proceso de aprendizaje), la gran mayoría ha valorado positivamente esta relación.

- En el segundo bloque de contenidos (conocer las necesidades del alumnado en la materia de L. Castellana), la gran mayoría ha valorado positivamente esta relación.

- En el tercer bloque de contenidos (motivación), el 100\% muestra una actividad motivadora y gratificante.

\section{Conclusiones de la experiencia}

La propuesta de uso de Socrative en este proyecto ha resultado excelente, tanto por su facilidad de uso, las posibilidades que ofrece a nivel educativo en el que nos encontramos, la posibilidad inmediata de conocer a todos los participantes una percepción de su autoevaluación de sus conocimientos de forma inmediata y lúdica, favorece la competitividad y genera un espíritu de esfuerzo por alcanzar el éxito, en el proceso de enseñanza y aprendizaje. Las TIC son en general una herramienta que facilita el proceso de enseñanza y aprendizaje pero su uso en el aula requiere una metodología adecuada y un cambio metodológico notable.

Los resultados obtenidos en el cuestionario final y la observación directa realizada durante el desarrollo de las actividades, y posteriormente mostrada a través del diario, ponen de manifiesto que la satisfacción del alumno y su interés mostrado con respecto al aprendizaje de lengua en este tipo de entorno es altamente satisfactoria. Las opiniones de los alumnos expresan una elevada sensación de haber mejorado el aprendizaje y entendido mejor los conceptos de lengua, con el uso de este tipo de herramientas.

Para llegar a esta conclusión se han utilizado los tres instrumentos de recopilación de información (test de evaluación, diario, y el cuestionario). Los test de evaluación no reflejan una evaluación en el aprendizaje como tal pero, como ya se comentó en apartados anteriores, la motivación es fundamental para el 
mismo y, tanto el diario de clase como el cuestionario final manifiestan un claro interés de los alumnos(as) en el uso de las TIC.

La experiencia realizada también ha mostrado algunos obstáculos, que han impedido en mayor o menor medida el correcto desarrollo del proyecto en toda su extensión. Dada la importancia de las mismas, me ha parecido conveniente incluir éstas a continuación:

- En primer lugar cabe mencionar la escasa disponibilidad de espacio y de recursos tecnológicos (Tablet $\mathrm{u}$ ordenadores), en el aula de informática que pudiera solventar la ausencia del dispositivo móvil entre muchos de ellos en el proyecto. En segundo lugar, el mal funcionamiento de Internet el algunas ocasiones provocó la anulación de la actividades.

- Otra limitación importante a considerar es el efecto novedad, con lo que no podemos cuantificar los efectos del mismo sobre los alumnos(as), ni sobre sus tiempos de aprendizaje.

- Para finalizar, mencionar, la corta duración del proyecto, debido a las características en las que se encuentra enmarcado el mismo.

Con todo lo anterior y, principalmente debido a que el tiempo y los recursos empleados son limitados, la calidad de la investigación ha podido verse influenciada negativamente.

Los test de evaluación al finalizar cada una de las unidades didácticas han sido claramente motivadores para los alumnos(as), a la par que han sido capaces de crear competitividad entre ellos; sin embargo considero más conveniente para futuras ocasiones realizar un test de autoevaluación, que les dé la posibilidad de repetirlo si así desean y aprender de esta forma de sus propios errores.

Igualmente, el proyecto realizado se ha mostrado capaz de prestar apoyo suficiente a aquellos alumnos con necesidades educativas específicas o con distintos ritmos de aprendizaje, a través de los múltiples formatos de formular las preguntas, así como, permite conocer una percepción previa de los conocimientos asimilados por los estudiantes, y que en muchas ocasiones el tutor-docente es incapaz de conocer, pues muchos de ellos no se manifiestan en el aula para solicitar ayuda hacia sus desconocimiento de conceptos de lengua por vergüenza, timidez, entre otras causas, es por ello, que con esta práctica motivadora y lúdica el tutor-docente podrá hacer modificaciones y mejoras en el afianzamiento de la formación de conocimiento de lengua, en el proceso de formación de enseñanza y aprendizaje. El descubrimiento de una nueva actividad, dado el carisma innovador que para ellos presentaba este proyecto, se imponía siempre frente al aprendizaje de los pasos previos. Se plantea así la posibilidad de usar este tipo de herramientas potenciando la capacidad de adaptación a las necesidades que presenten los alumnos.

Para garantizar que todos los alumnos(as) alcancen los objetivos mínimos se podrían plantear diferentes niveles de formularios en línea de forma que el paso de un nivel a otro sea mediante algún tipo de actividad de acceso al siguiente nivel. Así como, de organizar grupos para heterogéneos donde a través del juego, por ejemplo: "las carreras espaciales" que ofrece Socrative, les permita interactuar entre ellos, colaborar, y ayudar a los que tienen más dificultad en el aprendizaje. En la red existen multitud de actividades que se pueden aplicar a nuestro proyecto, así como herramientas de fácil uso para crear nuestras propias tareas.

Dado el tipo de alumnado con el que se ha planteado la experiencia me parece relevante la disponibilidad de un ordenador u Tablet por alumno(a). En el proyecto actual se permitió a los alumnos que trajesen sus propios dispositivos móviles, ya que el aula de informática utilizada carece de ellos, pero muy pocos participaron en esta iniciativa. Además, no se debe abusar de las actividades largas y de excesiva dificultad, pues hacen que los alumnos pierdan interés y motivación por la materia. En este sentido, los cuestionarios cortos, y bien estructuradas son más productivos que muchos ejercicios largos, cuya realización se torna un proceso tedioso para los alumnos (as). En esta misma línea, las cuestiones deben ser variadas, para que el estudiante obtenga una mirada diferente y los profesores podamos observar el proceso que ello conlleva.

La inclusión de cuestionarios grupales y que fomenten la competitividad también puede ser considerada como una propuesta de mejora para ocasiones posteriores. Este tipo de actividades fomentan el trabajo colaborativo y hacen que los propios alumnos manifiesten un mayor interés al tener que "enfrentarse" a sus compañeros.

Este proyecto no ha pretendido en ningún momento que todos los estudiantes se convierten en apasionados de la lengua, pero sí que la miren desde un prisma diferente y se convierta en elemento motivador para su propio aprendizaje.

Finalmente, destacar que los errores detectados por parte del docente (tanto en el desarrollo del proyecto y su puesta en funcionamiento), como por parte del alumnado (en el trabajo a diario), deberían ser 
utilizados como trampolín para futuros proyectos. Estos errores se deben tener presentes a la hora de planificar futuras actividades y en la selección de los distintos recursos.

\section{Referencias}

Borthwick, A. y Lobo, I. (2005). Lessons from Costa Rica. Learning and Leading with Technology, 33(2), 1821.

Charman, D. (1999). Issues and Impacs of Using Computer-Based Assessments (CBAs) for Formative Assessment. En S. Brown, P. Race, y J. Bull (eds.), Computer-Assissted in Higher Education. (pp. 85-94). London: Kogan.

Condie, R. y Livingston, K. (2007). Blending online learning with traditional approaches: changing practices [versión electrónica]. British Journal of Educational Technology, 38, 337-348.

Condie, R. y Munro, B. (2007). The Impact of ICT in Schools: a landscape review. UK: Becta.

García Ramos, J. M. (1989). Bases pedagógicas de la evaluación. Guía práctica para educadores. Madrid: Síntesis.

García, J., Pérez, M. J., Rodríguez, B., y Sánchez, M. C. (2002). Evaluar en la red. Revista de Teoría de la Educación. Educación y cultura en la sociedad de la información, 3. Disponible en: http://www3.usal.es/ teoriaeducacion/rev_numero_03/n3_art_sanchez-rodriguez.htm

Lara, S. (2001). La evaluación formativa en la Universidad a través de Internet: aplicaciones informáticas y experiencias prácticas. Pamplona: Eunsa, Ediciones de la Universidad de Navarra.

Lara, S. (2003). La evaluación formativa a través de internet. En M. Cebrián, Enseñanza virtual para la innovación universitaria (pp. 105-117). Madrid: Narcea.

Passey, D. y Rogers, C., (2004). The motivational Effect of ICT on Pupils. Departament of Educational Research Lancaster University: University of Lancaster [en línea] Disponible en: http://portaldoprofessor.mec.gov.br/storage/materiais/0000012854.pdf.

Taras, M. (2003). To feedback or Not to Feedback in Student Self-assessment, Assessment \& Evaluation in Higher Education, 28(5), 549-565.

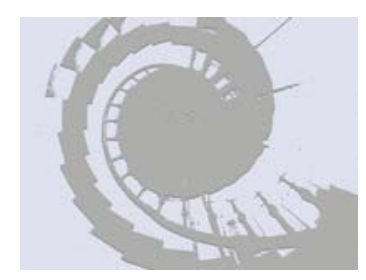

